\title{
Gamma-modulated Wavelet Model for Internet of Things Traffic
}

\author{
Yuhong Li, Yuanyuan Huang \\ State Key Laboratory of Networking and \\ Switching Technology \\ Beijing University of Posts and \\ Telecommunications \\ Beijing, China
}

\author{
Xiang Su, Jukka Riekki, Huber Flores Chao Sun, Hanyu Wei, Hao Wang, Lei Han \\ Centre for Ubiquitous Computing \\ University of Oulu \\ Oulu, Finland \\ Network Technology Laboratory \\ Huawei Technologies Co., Ltd. \\ Nanjing, China
}

\begin{abstract}
Promoted by sensor, big data and mobile computing technologies, the number of Internet of Things (IoT) applications and services is increasing rapidly. The massive amounts of heterogeneous data produced by a large variety of IoT devices require us to re-think its influence on the network. In this paper, we study the characteristics of IoT data traffic in the context of smart city. We generate data traffic according to the characteristics of different IoT applications. We propose a Gamma modulated wavelet method for statistical characterization of both IoT data and the aggregated traffic, aiming at analyzing the influence of IoT data traffic on the access and core network. By using Gamma function to modulate the coefficients of the wavelet, both the long range and short range dependency of the IoT data traffic can be described through fewer parameters. The Gamma modulation also reduces the independency of the coefficients and improves the accuracy of the Wavelet model.
\end{abstract}

Keywords-Internet of Things; traffic modelling; wavelet;

\section{INTRODUCTION}

Internet of Things (IoT) connects devices producing massive amounts of heterogeneous data. IoT applications and services have been developed and introduced to our daily life in domains like healthcare, goods tracking and industrial process monitoring. With the increase of the number of applications and devices, large amounts of IoT data traffic will be generated and delivered to the access and core networks, which will influence other applications and services currently supported by the networks, such as voice and data services. Moreover, the data traffic produced by IoT applications varies greatly, from several bits generated by simple sensors to high quality video clips of several hundred megabytes generated by video cameras. Also, the pattern of data generation varies from periodic to sporadic and the same application can produce different amounts of data traffic on different platforms. Thus, it is difficult to predict the influence that IoT data traffic has on the public networks and infrastructure facilities.

Deep understanding of the characteristics of IoT applications and their data traffic is crucial to accurately analyze and estimate the performance of the network and the applications. Network operators can utilize such knowledge to optimize their network infrastructure, components, services, and costs. Traffic engineering and management tasks can be enhanced as well.
Traffic modelling is an efficient approach for describing the characteristics of network applications [1-3]. However, the current traffic models are based on Internet applications. Traffic modelling for IoT applications presents considerable challenges not targeted in the previous research. First, contrast to the traditional network applications that interact with humans, IoT applications use large numbers of devices providing data in heterogeneous and complex environment and situations. Second, different data analysis and processing techniques, such as semantic analysis and data mining, results in different types of data, from raw data to aggregated objects, semantic information, and actionable knowledge. This leads to more complex characteristics of data traffic. Moreover, the future IoT will need support from cloud computing and big data analysis technologies. These technologies will also influence heavily data traffic. Third, it is becoming a consensus that future IoT will have horizontal architecture [4], integrating various open systems, environments and platforms. How these environments and platforms will influence the traffic is still unclear.

In this paper, we analyze and summarize the features of IoT data and the traffic resulting from transferring this data. We have collected IoT data from a simulated environment which produces IoT data according to some existing research results. We present the analysis of the statistical characteristics of this data. Based on the analysis, we propose a Gamma modulated wavelet method to model the characteristics of the data. We also analyze the relationship between data traffic and the parameters of the Gamma model. The rest of the paper is organized as follows. We discuss related work in Section II, and summarize traffic characteristics of IoT applications in Section III. We elaborate the Gamma-modulated wavelet model for IoT traffic in Section IV, and then conclude the paper with discussing future research work in Section V.

\section{FROM MODELLING INTERNET TRAFFIC TO MODELLING IOT TRAFFIC}

\section{A. Models of Internet Traffic}

The large amount and varieties of services and applications in IP networks and their performance requirements on the network have made IP traffic modelling an active research topic. Some studies have exhibited that IP traffic has the characteristics of self-similarity and long-range dependence (LRD) [1-3], and peculiar behaviours have a significant impact 
on network performance. Real network traffic is different from that predicted by traditional and Markovian models. The multifractal property of network traffic is shown in [5-6]. Based on this finding, L-system models [7] and random cascades [8] have been proposed to describe the scaling behaviour of IP traffic. Furthermore, several ingenious and analytically simple procedures have been developed based on Markovian model that consider the peculiar behaviours [9-11], and hence can be used for calculating network performance and predicting future network traffic. For example, work in [11] has modelled collected M2M traffic with multi-state Markov Modulated Poisson Process (MMPP-n). The authors introduced an MMPP traffic model with hierarchical structure to approximate accurately the LRD of Internet traffic traces.

Many studies on the features of network traffic have demonstrated that the network traffic shows obvious scaling characteristics in a real environment. Wavelet transformation has the inherent capability for scale analysis, and has therefore been used to model network traffic, such as the Waveletdomain Independent Gaussian (WIG) [12] and Multi-fractal Wavelet Model (MWM) [5]. For instance, MWM combines the power of multifractals with the efficiency of the wavelet transform in a flexible framework natural for characterizing and synthesizing positive LRD data. The authors use a simple multiplication to the coefficients to set a simple constraint, thus the positive process can be guaranteed. This makes MWM suitable for modelling positive, stationary, and LRD data, and hence suitable to the analysis and synthesis of network traffic.

\section{B. IoT Traffic Modelling: State of the Art}

Traffic modelling has been studied in the context of machine-to-machine (M2M) and ubiquitous sensor networks (USN). In [13], Poisson arrival process was assumed as the traffic model of each individual sensor node. The On/Off model for USN traffic models is analyzed in [14]. The authors proved that On period distribution and Off period distribution could be described by generalized Pareto distribution. In [15], the autocorrelation functions for electrocardiogram and body temperature monitoring traffic were studied (both nonPoisson). The pseudo LRD traffic model was proposed in [16] for mobile sensor networks. [17] presents how the traffic flows for fixed and mixed fixed/mobile sensor nodes have the self-similar nature with middle level of self-similarity. The traffic flow for reconfiguration and signalling has self-similar nature with high level of self-similarity.

Work in [18] compared M2M and smartphone traffic in several aspects including temporal traffic patterns, device mobility, application usage and network performance. By analysing a week long traffic trace collected from a cellular service provider's core network, the authors show that M2M traffic typically exhibits different diurnal patterns, and M2M more likely generates synchronized traffic resulting in bursty aggregate traffic volumes compared to smartphones. Wavelet transformation was used to decompose device model and subscriber time series and therefore the time series were grouped into distinct clusters. However, only the similarity score between time series was analyzed and computed.

In particular, in work [19], a framework for modelling
M2M traffic model, Heterogeneous Chain Modulated Generic Process (HCMGP), is presented. The authors use wavelet scalograms to perform M2M traffic analysis, which described the signal energy on a time domain, in order to identify the features of different traffic. They decompose the traffic process through wavelet to obtain the wavelet coefficients to construct the wavelet scalograms. We share the same idea with this work in using wavelet transform. However, we decompose the probability density of various types of traffic and the aggregated traffic, and use the parameters of Gamma distribution to model the level of the similarity of IoT traffic.

In general, the traffic model of a single application type has been considered in the context of M2M, such as telemetry [18], video surveillance [20], and YouTube [21]. To the best of our knowledge, we have not found any works discussing the model of aggregated IoT traffic, though this is important for network operators to optimize their network

Due to the complexity introduced by IoT applications and their environments and platforms, the current data traffic modelling methods cannot be used directly for IoT applications in IoT environment. In this paper, we present a Gamma modulated Wavelet model with shape parameter $m$ and scale parameter $n$ (GMW-mn) to describe the characteristics of IoT traffic, which reflect also the level of similarity of the IoT traffic and the scale of the IoT network.

\section{TYPICAL IOT APPLICATIONS AND TRAFFIC CHARACTERISTICS}

\section{A. Typical IoT Applications}

Many IoT applications have been developed for a wide range of domains. In general, the devices, such as various types of sensors, are connected to the Internet through sinks and gateways. Unlike traditional communication scenarios where human is in the centre of the information exchange, machines play an important role in IoT communications. Typical IoT applications form the following three categories:

1) Machine-to-machine, where no human interaction is involved. For example, remote sensors send measurement results to a sink or a gateway;

2) Human-to-machine, where an operator is involved in configuring, supervising and operating devices remotely;

3) Machine-to-human, where devices collect data and send them to human users. For example, a vehicle initiates a call with an emergency service when an accident has occurred.

Category 2 typically generates much downlink traffic that is similar to the current Internet traffic. Category 3 typically generates much uplink traffic. Due to the data processing, including mining and reasoning functions in the networks, the uplink and downlink data traffic created by this type of applications is similar to the traffic in the current Internet networks. Therefore, in this paper we concentrate on analysing the data traffic generated by the machine to machine applications (Category 1).

\section{B. Characteristics of IoT Traffic}

The most important characteristics of IoT traffic is that it is 
independent from human behaviour. In particular, the timing of the information exchange is no longer defined by humans. In general, the IoT traffic may include the following data in smart city use case:

- Heart beat, carrying information about the status of the node itself. Heart beat is normally triggered by the devices in order to stay connected with the infrastructure. Therefore, this type of data traffic has regular traffic pattern, and has a constant size of data.

- Event trigger, normally initiated by the server side to trigger an action of an M2M device, for example, to report the humidity of a room to the network. The trigger process is irregularly generated by the servers.

- Payload data, used to exchange payload between devices and servers in the networks. Data traffic is variable and can either be of constant size like in telemetry, or of variable size like images in video surveillance.

- Node update, used to maintain the normal operation of devices, for example, a server in the network may push configurations or firmware to devices, and the nodes will reboot running the update from the servers.

Due to the above mentioned IoT data traffic, few short packets may be transmitted per device, and may be at low duty cycle. The traffic pattern created by a single device may have small variations, however, there are massive number of devices in the IoT network. Generally, the IoT traffic has the following characteristics:

- The traffic varies heavily with the application scenarios. In the same application scenario, the traffic varies also with IoT platforms and the number of IoT devices.

- The traffic model for a single scenario might be simple. However, when multiple scenarios are integrated together the traffic model becomes complicated. This results in an unknown influence on the carrier networks.

- New applications and scenarios may emerge frequently with maturing IoT related technologies. This may change the total IoT traffic in an unexpected way.

Hence, IoT traffic can no longer be modelled and predicted by traditional approaches and a new modelling paradigm needs to be developed.

\section{AN WAVELET MODEL BASED ON GAMMA DISTRIBUTION}

\section{A. Traffic Simulation and Collected Results}

In order to characterize the aggregated IoT traffic from different scenarios, we have generated a large set of IoT traffic by simulating different scenarios in the network simulator NS3 [22]. Fig. 1 illustrates the network configuration we used, which is based on the smart city scenario [23], where different applications with different data traffic patterns are involved. To be able to obtain the aggregated traffic at different levels, we designed eight subnetworks, each with gateway (we call a gateway at the subnetwork level as a sink). The traffic from each sink is aggregated at the gateways which connect to the Internet through another gateway. To produce as realistic IoT data as possible, we generate the data according to characteristics revealed by other researchers.

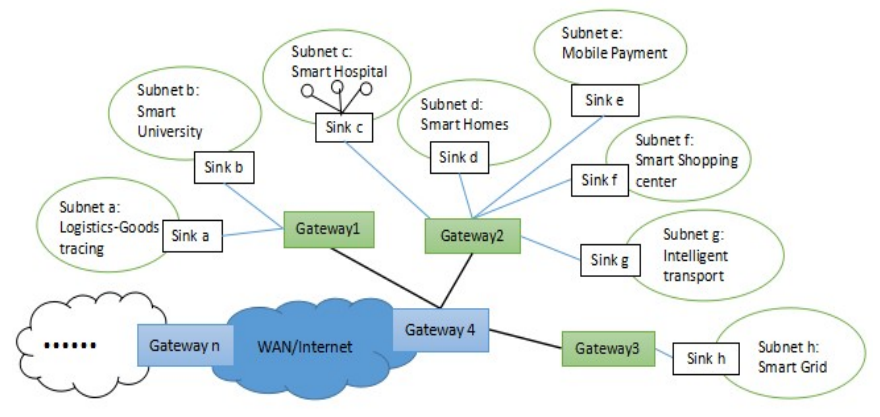

Fig. 1. Network architecture for collecting data

\section{(a) Logistics goods tracking [14]}

The logistics goods tracking follows the On-Off model, where the On-Off time follows Pareto distribution. Packet intervals follow the normal distribution with parameters of $(0.2,1)$ during the On time (Table I). Data from 800 goods tracking are collected, with random On and Off time.

TABLE I. DATA PATTERN OF LOGISTICS GOODS TRACKING

\begin{tabular}{|l|l|l|l|}
\hline & Pareto distribution & Packet size & Packet intervals \\
\hline On time & $(0.60,19.654,4.99)$ & 50 bytes & Normal distr. $(0.2,1)$ \\
\hline Off time & $(0.009,10.026,0.001)$ & N.A. & N.A. \\
\hline
\end{tabular}

\section{(b) University Campus [24]}

As shown in Table II, there are five types of sensors with different numbers serving for different purposes in universities. Three types of sensors, i.e., humidity detector, temperature sensor, and smoke detector, generate data packets periodically. The surveillance video camera generates packets every 15 seconds, and the packet lengths follow the logarithmic distribution. The packet length of each e-card check-in service is 205 bytes. However, the interval of check-in services follows normal distribution. In addition, altogether data from 15 universities are produced.

TABLE II. IOT DEVICES AND TRAFFIC PATTERN OF EACH UNIVERSITY

\begin{tabular}{|c|c|c|c|}
\hline Device type & $\begin{array}{c}\text { Device } \\
\text { number }\end{array}$ & Packet size (byte) & $\begin{array}{c}\text { Period (s)/ } \\
\text { Intervals }\end{array}$ \\
\hline Humidity detector & 50 & 90 & 1800 \\
\hline Temperature sensor & 50 & 80 & 600 \\
\hline Smoke detector & 60 & 80 & 600 \\
\hline $\begin{array}{c}\text { Surveillance video } \\
\text { (security) }\end{array}$ & 45 & $\begin{array}{c}\text { Lognormal } \\
\text { distr.(5.9,1.2) }\end{array}$ & 15 \\
\hline e-card check-in & 1050 & 205 & Normal distr. \\
\hline
\end{tabular}

\section{(c) Smart Hospital [25]}

The sensors and traffic patterns of equipments in a hospital is illustrated in Table III. There are 50 equipments in each hospital, and data from 10 hospitals are generated.

TABLE III. SENSORS AND TRAFFIC PATTERN IN EACH HOSPITAL

\begin{tabular}{|c|c|c|}
\hline Device type & Packet size (byte) & Period (s) \\
\hline cardiograph & 125 & 0.2 \\
\hline Blood pressure & 125 & 0.5 \\
\hline Pulse & 125 & 0.2 \\
\hline Body temperature & 27 & 5 \\
\hline
\end{tabular}

\section{(d) Smart homes [26]}

The traffic patterns of each household is shown in Table IV. Altogether data from 100000 households are generated. 
TABLE IV. IOT DEVICES AND TRAFFIC PATTERN OF EACH HOUSEHOLD

\begin{tabular}{|c|c|c|c|}
\hline Device type & Device number & Packet size (byte) & Period (s) \\
\hline Humidity detector & 5 & 90 & 1800 \\
\hline Temperature sensor & 4 & 80 & 600 \\
\hline Smoke detector & 2 & 80 & 600 \\
\hline Light switch & 5 & 100 & 1800 \\
\hline Sensor for security system & 1 & 80 & 600 \\
\hline Electric meter & 1 & 90 & 600 \\
\hline
\end{tabular}

(e) Mobile Payment [27]

As shown in Table $\mathrm{V}$, the data packet among payments follows exponential distribution. 2050 payments are collected.

TABLE V. TRAFFIC PATTERN OF EACH PAYMENT THROUGH MOBILE

\begin{tabular}{|l|c|c|}
\hline & Packet size (byte) & Packet intervals \\
\hline Data type & 205 & Exp. distribution $(\mu=2.5 \mathrm{~s})$ \\
\hline
\end{tabular}

\section{(f) Smart Shopping Centre [28]}

Devices and traffic data of each shopping centre is shown in Table VI. The data intervals of payment and retail system follows exponential and Pareto distribution respectively. Data of 8 shopping centres are produced.

TABLE VI. DEVICES AND TRAFFIC PATTERN OF EACH SHOPPING CENTRE

\begin{tabular}{|l|l|l|l|}
\hline Device type & $\begin{array}{l}\text { Device } \\
\text { number }\end{array}$ & $\begin{array}{l}\text { Packet size } \\
(\text { byte }) / \text { distr. }\end{array}$ & Period(s)/Interval \\
\hline Humidity detector & 20 & 90 & 1800 \\
\hline Temperature sensor & 10 & 80 & 600 \\
\hline Smoke detector & 25 & 80 & 600 \\
\hline $\begin{array}{l}\text { Surveillance } \\
\text { (security) }\end{array}$ & 20 & $\begin{array}{l}\text { Log normal } \\
\text { distr. }(5.9,1.2)\end{array}$ & 15 \\
\hline Payment & 10 & 205 & Exp. distr. $(\mu=2.5$ sec. $)$ \\
\hline Retail system & 10 & 72 & Pareto distr. $(1,10)$ \\
\hline
\end{tabular}

\section{(g) Intelligent Transport [29]}

The traffic data produced by each vehicle is illustrated in Table VII. Each vehicle produces data at an interval following the normal distribution. Altogether 1000 vehicles producing data with different starting time are collected.

TABLE VII. TRAFFIC PATTERN OF EACH VEHICLE

\begin{tabular}{|l|l|l|}
\hline & Packet size (byte) & Packet intervals \\
\hline Data type & 149 & Normal distribution $(80,8)$ \\
\hline
\end{tabular}

\section{(h) Smart Grid [18]}

The data traffic caused by one voltage transformer follows On-Off model (Table VIII). Totally data from 600 transformers are collected, with random On and Off times.

TABLE VIII. TRAFFIC PATTERN CAUSED BY ONE VOLTAGE TRANSFORMER

\begin{tabular}{|l|l|l|l|}
\hline & Time $(\mathrm{min})$ & packet size(byte) & Packet intervals \\
\hline On & 5 & 135 & Exp. distr. $(\mu=60 \mathrm{~s})$ \\
\hline Off & 15 & N.A. & N.A. \\
\hline
\end{tabular}

Fig. 2 illustrates data collection at the corresponding Sink and Gateway 1-4 during a period of 6000 seconds.

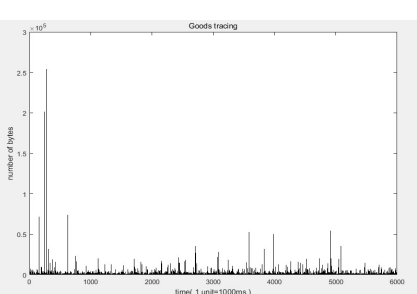

(a) Goods trackings

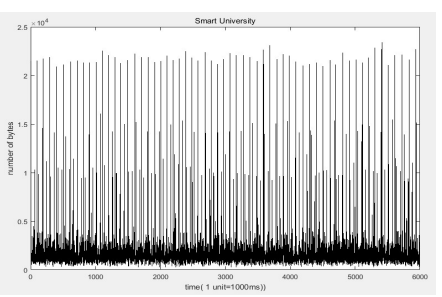

(b) University campus

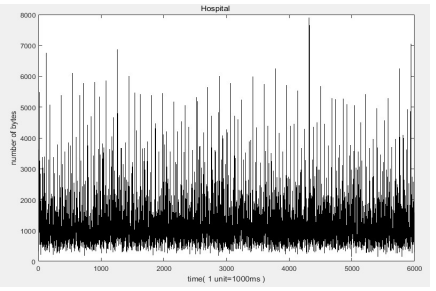

(c) Smart hospitals

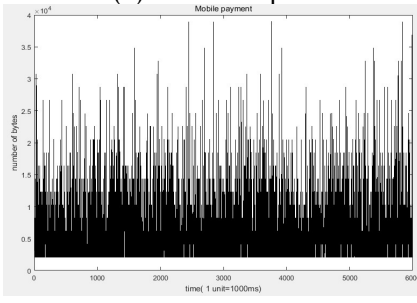

(e) Mobile payments

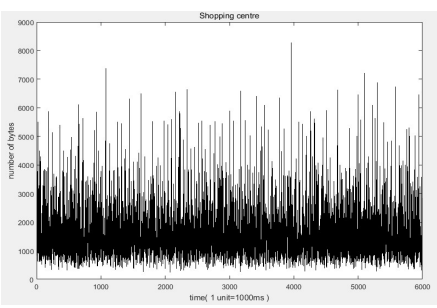

(g) Intelligent transports

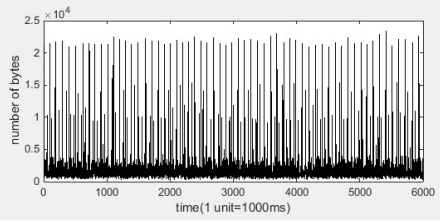

(i) Gateway 1

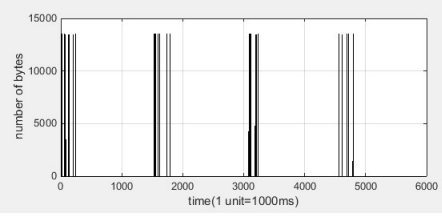

(g) Gateway 3

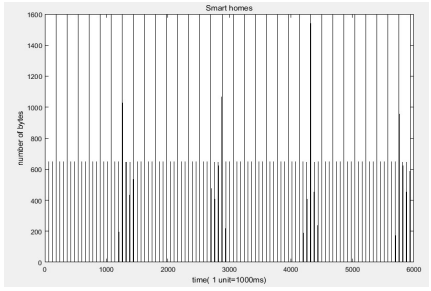

(d) Smart homes

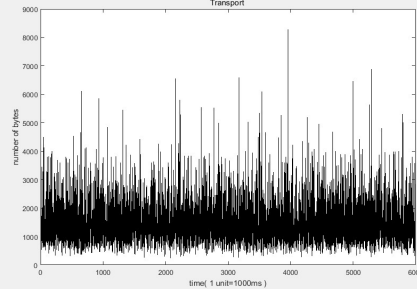

(f) Smart shopping centres

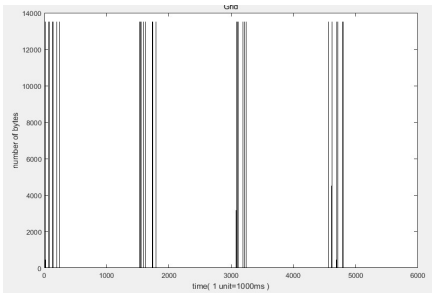

(h) Smart grids

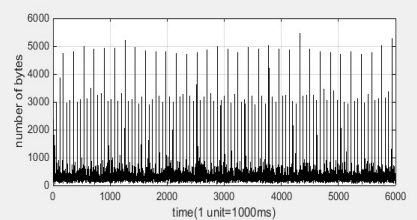

(j) Gateway 2

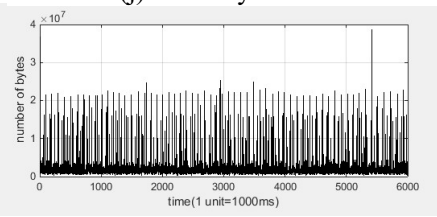

(h) Gateway 4
Fig. 2. Amount of data at sinks and gateway for 6000 seconds

\section{B. A Gamma Modulated Wavelet Model}

We start the analysis by estimating the Probability Density Function (PDF) of the traffic. We use ksdensity function (Kernel smoothing function estimate) [30] to estimate the probability density of the sampled data at the given time point of each sink, and at the gateway. Namely, let $\left(x_{1}, x_{2}, \ldots, x_{n}\right)$ be an independent and identically distributed sample drawn from some distribution with an unknown density $f$. Its kernel density estimator is:

$$
\hat{f}_{h}(x)=\frac{1}{n} \sum_{i=1}^{n} K_{h}\left(x-x_{i}\right)=\frac{1}{n h} \sum_{i=1}^{n} K\left(\frac{x-x_{i}}{h}\right)
$$

where $K(\bullet)$ is the kernel, a non-negative function that integrates to one and has mean value of zero; $h>0$ is a smoothing parameter.

Second, we perform Discrete Wavelet Transform (DWT) 
[31] on the PDF of the sampled data. Wavelet bases have the desirable property of being able to approximate a large class of functions. Moreover, they are able to achieve good global approximation properties due to their locally compact nature. This allows us to model the PDF of IoT traffic that may contain bumps and/or abrupt variations.

Similar to the work in [32], we represent the density as a linear combination of wavelet bases, namely

$$
p(x)=\sum_{j_{0}, k} U_{j_{0}, k} \phi_{j_{0}, k}(x)+\sum_{j \geq j_{0}, k}^{\infty} W_{j, k} \psi_{j, k}(x)
$$

where $\mathrm{x} \in \mathfrak{R}, p(x)$ is the PDF of the sampled data. $\phi(x)$ and $\psi(x)$ are the scaling and wavelet basis functions respectively, and $U_{j_{0}, k}$ and $W_{j, k}$ are scaling and wavelet basis function coefficients.

$$
\begin{aligned}
& \phi_{\mathrm{j}_{0}, k}(x)=2^{j_{o} / 2} \phi\left(2^{j_{o}} x-k\right) \\
& \psi_{j, k}(x)=2^{j / 2} \psi\left(2^{j} x-k\right)
\end{aligned}
$$

We assume $\phi(x)$ and $\psi(x)$ and their scaled and translated versions form orthogonal bases for their respective spaces. Therefore, given $N$ samples, the above coefficient can be approximated as the sample average, namely

$$
U_{j_{0}, k}=\frac{1}{N} \sum_{\mathrm{i}=1}^{N} \phi_{j_{0}, k}\left(x_{i}\right)
$$

$\phi(x)$ can be found by numerically solving the dilation equation and $\psi(x)$ can then be directly obtained by solving the wavelet equation. The dilation equation is given by:

$$
\phi(x)=2 \sum_{\mathrm{k}} l(k) \phi(2 x-k)
$$

where $l(k)$ are the low-pass filter coefficients associated with a particular scaling function family. This can be numerically solved using iterative procedures. After obtaining $\phi(x), \psi(x)$ can be obtained by using the high pass filter coefficients $h(k)$ and solving the wavelet equation:

$$
\psi(x)=2 \sum_{k} h(k) \phi(2 x-k)
$$

Third, based on many observations, we use the Gamma function [33] with different parameters to fit the scaling coefficient $U_{j_{0}, k}$, and polynomial to fit the wavelet coefficient $W_{j, k}$ of the probability density function $p(x)$. This is also because Gamma distribution can offer more flexibility parameterization than other kinds of heavy-tailed density for wavelet empirical histograms characterization, namely the distribution $f(x)$ can be given by (7)

$$
\begin{aligned}
& f(x)=\frac{1}{n \Gamma(m, n)}\left(\frac{x}{n}\right)^{m-1} e^{-x / n} \\
& \Gamma(m, n)=n^{m} \int_{0}^{\infty} x^{m-1} e^{-x n} d x
\end{aligned}
$$

where $m$ is the shape parameter and $n$ is the scale parameter, $\Gamma(\bullet)$ is the Gamma function.

Fig. 3 illustrates the results of $U_{j_{0}, k}$ and $W_{j, k}$, and the corresponding results. Due to the space limitation, we show only the results at the Gateway 2 and 4.

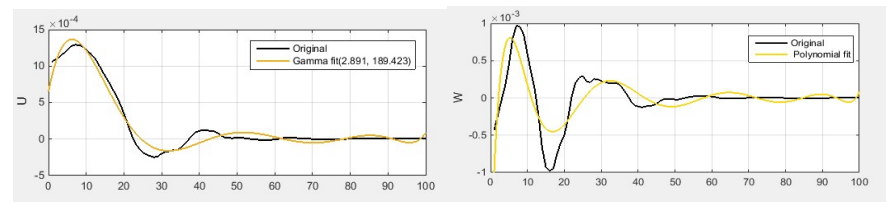

(a) $\mathrm{U}_{\mathrm{j} 0, \mathrm{k}}$ and $\mathrm{W}_{\mathrm{j}, \mathrm{k}}$ at Gateway 2

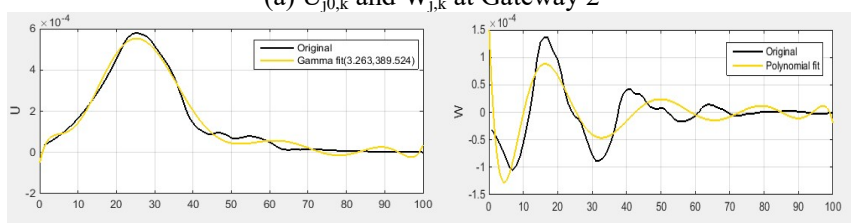

(b) $\mathrm{U}_{\mathrm{j} 0, \mathrm{k}}$ and $\mathrm{W}_{\mathrm{j}, \mathrm{k}}$ at Gateway 4 (Total data)

Fig. $3 U_{j 0, k}$ and $W_{j, k}$ of the wavelet transform of PDF at Gateway 2 and 4 and the corresponding fitting results.

Note that as an approximating signal, $U$ includes almost all the information of the original function, while $W$ describes the details in each scale. Therefore, we select polynomial function to fit $W$ to keep the model as simple as possible.

\section{Analysis}

To show our modelling results, we re-construct the PDF function based on the Gamma modulated wavelet scaling coefficient $U$ and the polynomial fitted wavelet coefficient $W$. Fig.4 illustrates the results of the original and re-constructed PDF at each gateway.

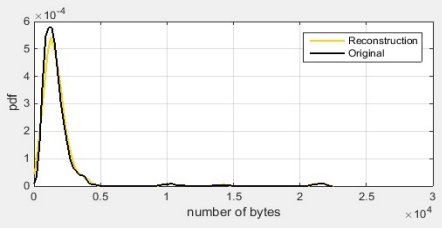

(a) PDF at Gateway 1

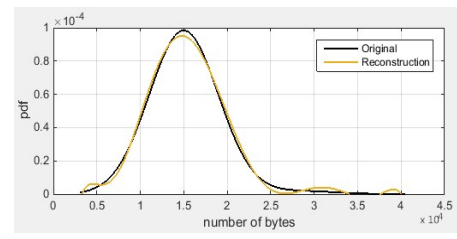

(c) PDF at Gateway 3

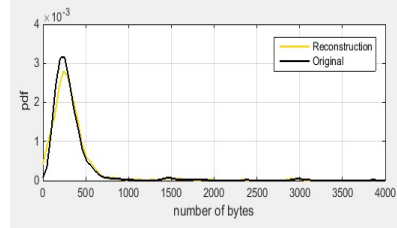

(b) PDF at Gateway 2

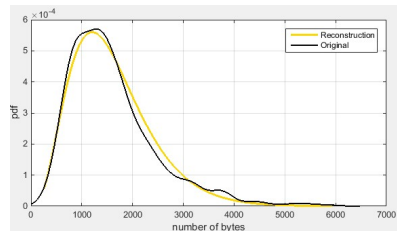

(d) PDF at Gateway 4

\begin{tabular}{|c|c|c|c|c|c|c|}
\hline No & Applications & $\begin{array}{c}\text { Hurst } \\
(H)\end{array}$ & $m$ & $n$ & $\begin{array}{c}\text { Aggregated } \\
H, m, n\end{array}$ & $\overline{\text { LRD }}$ \\
\hline (a) & Goods tracking & 0.148 & 1.89481 & 194.308 & \multirow{2}{*}{$\begin{array}{l}H=0.169 \\
m=2.08128 \\
n=349.308\end{array}$} & \multirow{2}{*}{$\begin{array}{l}\text { very } \\
\text { week }\end{array}$} \\
\hline (b) & $\begin{array}{l}\text { University } \\
\text { Campus }\end{array}$ & 0.208 & 2.18369 & 335.008 & & \\
\hline (c) & Smart Hospital & 0.558 & 2.46554 & 322.627 & \multirow{5}{*}{$\begin{array}{l}H=0.579 \\
m=2.89106 \\
n=689.894\end{array}$} & \multirow[t]{5}{*}{ week } \\
\hline (d) & Smart homes & 0.559 & 2.65739 & 398.524 & & \\
\hline (e) & Payment & 0.560 & 2.87645 & 216.692 & & \\
\hline (f) & ShoppingCentre & 0.600 & 2.93508 & 208.745 & & \\
\hline (g) & Transport & 0.602 & 3.46554 & 426.382 & & \\
\hline (h) & Smart Grid & 0.901 & 3.82789 & 188.644 & $H=0.901$ & strong \\
\hline
\end{tabular}

Fig. 4 Re-constructed PDF at each gateway.

From the results we can see that our proposed model can well approximate the original data traffic.

In order to estimate the self-similarity of the data set shown in Fig. 3, we use R/S method [34] to calculate the Hurst parameter. Table IX presents the results.

TABLE IX. HURST VALUE OF SERVICE DATA AND AGGREGATED DATA 
From Table IX we notice that the value of $m$ in the Gamma distribution is related with the level of self-similarity of the traffic data. $n$ is related with the number of the devices in the network, in other works, the total amount of the traffic data. In the PDF function, $n$ decides the location of the peak value of the traffic data.

\section{CONCLUSIONS AND FUTURE WORK}

In this paper, we present the results of modelling IoT data traffic by performing wavelet transform on the probability density of the traffic, and using the Gamma function to modulate the coefficients of the wavelet. We found that the parameters of the Gamma function can reflect both the selfsimilarity and the scales of the IoT data and network. By adjusting the parameters of the Gamma function, the probability density function of the IoT data traffic can be characterized. As future work, we model data generated by more complex network configurations, including the downlink configuration and software/firmware updating data, and collect data from real-world applications to verify our model.

\section{ACKNOWLEDGMENT}

The work is partly supported by Huawei HIRP program.

\section{REFERENCES}

[1] W. Leland, M. Taqqu, W. Willinger, and D. Wilson, "On the self-similar nature of Ethernet traffic (extended version)," IEEE/ACM Transactions on Networking, vol. 2, no. 1, pp. 1-15, Feb. 1994.

[2] M. Crovella and A. Bestavros, "Self-similarity in World Wide Web traffic: Evidence and possible causes," IEEE/ACM Transactions on Networking, vol. 5, no. 6, pp. 835-846, Dec. 1997.

[3] V. Paxson and S. Floyd, "Wide-area traffic, "The failure of Poisson modeling," IEEE/ACM Transactions on Networking, vol. 3, no. 3, pp.226-244, June 1995.

[4] Y. Li, X.Su, J.Riekki, T. Kanter and R. Rahmani, "A SDN-based Architecture for Horizontal Internet of Things Services," IEEE International Conference on Communications (ICC), 23-27 May 2016, Kuala Lumper. 2016:1-7.

[5] R. Riedi and J. V'ehel, "Multifractal properties of TCP traffic: a numerical study," Technical Report No 3129, INRIA Rocquencourt, France, Feb 1997, available at www.dsp.rice.edu/_riedi.

[6] R. Riedi, M. Crouse, V. Ribeiro, and R. Baraniuk, "A multifractal wavelet model with application to network traffic," IEEE Transactions on Information Theory, vol. 45, no. 4, pp. 992-1018, April 1999.

[7] P. Salvador, A. Nogueira, and R. Valadas, "Modeling multifractal traffic with stochastic L-Systems," in Proc. of GLOBECOM'2002, vol. 3, pp. $2518-2522$.

[8] A. Feldmann, A. Gilbert, and W. Willinger, "Data networks as cascades: Investigating the multifractal nature of internet WAN traffic," in Proc. of SIGCOMM, 1998, pp. 42-55.

[9] A. Andersen and B. Nielsen, "A Markovian approach for modeling packet traffic with long-range dependence," IEEE Journal on Selected Areas in Communications, vol. 16, no. 5, pp. 719-732, June 1998.

[10] P. Salvador, R. Valadas, and A. Pacheco, "Multiscale fitting procedure using Markov modulated Poisson processes," Telecommunications Systems, vol. 23, no. 1-2, pp. 123-148, June 2003.

[11] L. Muscariello, M. Meillia, M.Meo and et. al, "An MMPP-Based Hierachical Model of Internet Traffic," in Proc. of IEEE Internationcal Conference on Communications. pp.2143-2147, 2004.

[12] Ma S, Ji C. "Modeling video traffic in the wavelet domain," In Proc. of the 17th Annual IEEE Conf. on Computer Communication, INFOCOM. San Francisco: IEEE, 1998. 201-208.
[13] Obada Al-Khatib, Wibowo Hardjawana, Branka Vucetic, "Traffic Modeling for Machine-to-Machine(M2M) Last Mile Wireless Access Networks," Communications QoS, Reliability and Modelling Symposium 2014.

[14] Q. Wang and T. Zhang, "Source Traffic Modelling in Wireless Sensor Networks for Target Tracking," In Proc. of the 5th ACM International Symposium on Performance Evaluation of Wireless Ad hoc, Sensor and Ubiquitous Networks, Vancouver, Canada, October 27-31, 2008, pp.96100 .

[15] G. Messier and I.G. Finvers, "Traffic Models for Medical Wireless Sensor Networks". IEEE Communications Letters 2007, 11(1): pp.13-15.

[16] P. Wang, and I. F. Akyildiz, "Spatial Correlation and Mobility Aware Traffic Modelling for Wireless Sensor Networks". In Proc. Of IEEE GLOBECOM 2009, Honolulu, USA, Nov.30 - Dec. 4. 19(6):pp.1-6.

[17] A. Koucheryavy and A. Prokopiev, "Ubiquitous Sensor Networks Traffic Models for Telemetry Applications," in Proc. Of NEW2AN 2011, 6869: 287-294.

[18] M. Z. Shafiq, L. Ji and et.al. "Large Scale Measurement and Characterization of Cellular Machine-to-Machine Traffic", IEEE/ACM Transactions on Networking, 2013, 21(6):1960-1973.

[19] Z. M. Fadlullah, M. M. Fouda, N. K. A. Takeuchi, N. Iwasaki, and Y. Nozaki, "Toward intelligent machine-to-machine communications in smart grid". IEEE Communications Magazine, 49(4):60-65, 2011.

[20] I. Petiz, P. Salvador and A. Nogueira, "Characterization and Modeling of M2M Video Surveillance Traffic," in Proc. of the Fourth International Conference on Advances in Future Internet (AFIN 2012), pp.65-70.

[21] G. Horváth and P. Fazekas, "Characteriztion and Modelling of YouTube Traffic in Mobile Networks," in Proc. of the Fourteenth International Conference on Networks (ICN 2015), pp.115-121.

[22] NS-3: A Discrete-event Network Simulator. http://www.nsnam.org/.

[23] White paper, Standardization of Chinese Smart city, 2014. (in Chinese)

[24] L. Yong and G. Xiuchun, "Research of Pedestrian Traffic Characteristics in University Campus," ), in Proc. of The Fourth International Conference on Digital Manufacturing and Automation (ICDMA), Qingdao, 2013, pp. 1022-1024.

[25] A. Ahmad, A. Riedl, W. J. Naramore, N. Y. Chou and M. S. Alley, "Scenario-Based Traffic Modeling for Data Emanating from Medical Instruments in Clinical Environment," Computer Science and Information Engineering, 2009 WRI World Congress on, Los Angeles, CA, 2009, pp. 529-533.

[26] A. Orrevad, " M2M Traffic Characteristics-When machines participate in communication," Master of Science Thesis," Royal Institute of Technology, Sweden,01/12-2009.

[27] C. Zhang, H. Wang, Y. Cui, J. Wu and W. Wang, "TSWiFi: An optimal payment-based traffic sharing in mobile networks," IEEE International Conferenc on Broadband and Photonics, Bali, 2015, pp. 1-6.

[28] Osoba S B, "Appraisal of parking problems and traffic management measures in central business district in Lagos,", Journal of Sustainable Development, 2012, 5(8): pp.105-115.

[29] Drajic D, Krco S, Tomic I, et al, "Traffic generation application for simulating online games and M2M applications via wireless networks [C]," in Proc. of 9th Annual Conference on Wireless On-demand Network Systems and Services (WONS), 2012, pp.167-174.

[30] D. W. Scott, "Multivariate Density Estimation: Theory, Practice, and Visualization. New York: Wiley-Interscience," 2001.

[31] P. Flandrin, "Wavelet analysis and synthesis of fractional Brownian motion," IEEE Trans. on Information Theory, 1992, 38(2), pp.910-917.

[32] A. M. Peter, and A. Rangarajan, "Maximum Likelihood Wavelet Density Estimation With Applications to Image and Shape Matching," IEEE Transactions On Image Processing, Vol. 17, No. 4, pp.458-468, April 2008.

[33] E. W. Stacy, G. A. Mihram, "Parameter Estimation for a Generalized Gamma Distribution," Technometrics, 2012, 7(7), pp.349-358.

[34] B. Qian and K. Rasheed (2004), "Hurst exponent and financial market predictability IASTED," Financial Engineering and Applications (FEA 2004). pp. 203-209. 